Pteridines

Vol. 11, 2000, pp. $90-93$

\title{
Dihydroneopterin as a scavenger of nitrogen centered radicals
}

\author{
Karl Oettl, Joachim Greilberger and Gilbert Reibnegger
}

Institute for Medical Chemistry and Pregl-Laboratory,Karl-Franzens-University Graz, Harrachgasse 21II, A-8010 Graz, Austria

Running title: Dihydroneopterin and nitrogen radicals

This work was financially supported by the Austrian "Fonds zur Förderung der Wissenschaftlichen Forschung", project P12366-CHE.

\section{Summary}

Dihydroneopterin has recently been shown to either promote or decrease formation of free radicals or radicalmediated reactions, depending on the conditions. We icport here the scavenging activity of dihydroneopterin on nitrogen centered radicals. Diphenylpicrylhydrazyl (DPPH) and the radical cation of 2,2'-azino-di-[3ethylbenzthiazoline sulphonate] (ABTS) were used. Dinydroneopterin showed scavenging properties against either compound. In the case of DPPH radical scavenging by dihydroneopterin was comparable with trolox. In the ABTS system, dihydroneopterin had an even better c.nacity of radical scavenging as compared to trolox.

\section{introduction}

A number of papers published dining the pasi decade reported the interferince of pterins with free radical reactions $(1-17)$, like chemiluminescence of luminol (1, 2, 4, 5, 9, 10). LDL-oxidation (6), apoptosis (8), toxicity of hydrogen peroxide and chloramine $\mathrm{T}$ against bacteria $(3,11)$, nitration of tyrosine (14) or formation of reactive oxygen species (15-17). For both, radical scavenging and radical promoting activities, most studies were focussed on oxygen centered radicals. However, the reactivities of antioxidants with different radical species may vary considerably (18).

For a complete understanding of an in vivo situation concerning pterins and free radicals reactive nitrogen species have to be considered, too. For example, recently the interference of pterins with nitration of tyrosine by peroxynitrite has been reported (14). Moreover, nitrogen centered radicals are often used for the characterization of the antioxidant properties of compounds and biological fluids (19-24). We therefore tested the radical scavenging activity of dihydroneopterin with two systems frequently used for the measurements of antioxidative properties. Both assays are based on nitrogen-centered radicals and in
Joth assays trolox, a water soluble form of vitamin E, was used as a stmiard antioxidant.

Materal and Methods

Reagent:

7,8-Dihydroneopterin was obtained from Dr. Schircks laboratory (Jona, Switzerland). Diphenylpicrylhydrazyl (DPPH), trolox (6-hydroxy2,5,7,8-tetramethylchroman-2-carboxylic acid) and tris (tris-(hydroxymethyl)-aminomethane) were from Sigma (Vienna, Austria). All other reagents were obtained from E. Merck (Darmstadt, Germany).

\section{Scavenging of DPPH:}

The use of DPPH for the determination of antioxidants was first described by Blois (25). An ethanolic solution of DPPH $(100 \mu 1,300 \mu \mathrm{M})$ was mixed with increasing concentrations of dihydroneopterin and trolox, respectively, in $10 \mathrm{mM}$ Tris buffer, $\mathrm{pH}$ 7.4. The final solution had an ethanol concentration of $50 \%$. After 20 minutes at room temperature the absorbance at $517 \mathrm{~nm}$ was measured against $50 \%$ ethanol in Tris buffer as a blank (26).

Correspondence to: Dr.Karl Oettl, Institute of Medical Chemistry and Pregl-Laboratory,

Harrachgasse 21/II, A-8010 Graz, Austria

tel: ++4433163807544, fax: ++433163809610, e-mail: karl.oettl@kfunigraz.ac.at 
Scavenging of 2,2'-azino-di-[3-ethylbenzthiazoline sulphonate] (ABTS)

A commercial testkit, Randox Total Antioxidant Status, (Dr. Franz Tatzber KEG, Klosterneuburg, Austria) was used. The assay is based on the formation of the radical cation $\mathrm{ABTS}^{+}$, and its scavenging by antioxidants (19). The solutions of the testkit were prepared according to the instructions and contained chromogen, standard and substrate. The chromogen solution consisted of $6.1 \mu \mathrm{M}$ metmyoglobin and $610 \mu \mathrm{M}$ ABTS, substrate was $250 \mu \mathrm{M}$ hydrogen peroxide and as standard a solution of 1.6 $\mathrm{mM}$ trolox was used. The assay was not performed as a single time point but as a kinetic measurement and the lag time of the onset of radical formation was calculated $(19,27)$. For the assay $10 \mu 1$ of sample were mixed with $250 \mu \mathrm{l}$ chromogen solution in a cavity of a 96 well plate. To the mixture $50 \mu 1$ of substrate were added and the measurement of the absorbance at $620 \mathrm{~nm}$ was started immediately. From the intersection of the kinetic curves of blank (water) and standard the start time of the assay was calculated using a Microsoft Excel program. By this procedure different handling times of different plates are corrected. The lag times of samples were obtained by the same procedure and were compared to the values obtained using trolox. Two sets of experiments were performed in triplicate.

\section{Results}

The scavenging of DPPH radical by dihydroneopterin and trolox is shown in Fig. 1.

Both, dihydroneopterin and trolox gave a concentration-dependent decolorization of DPPH. The behaviour of dihydroneopterin is essentially the same, compared with trolox. In the experiment shown in the upper panel $50 \mu \mathrm{M}$ DPPH were used, and $100 \mu \mathrm{M}$ in the lower panel. In both cases maximum decolorization is achieved at about half the concentration of the antioxidant compared to DPPH. This is in accordance with earlier reports describing the antioxidant activity of trolox (26).

Using the ABTS assay we found a pronounced lag phase in radical formation when trolox was added to the assay mixture (Fig. 2, upper panel). After the lag phase radical production starts with the same rate as in the blank. When dihydroneopterin was used there was again a concentration-dependent lag phase followed by a radical formation rate as in the blank. The concentration dependence of the lag phase is shown in Fig. 2, lower panel showing straight lines for both trolox and dihydroneopterin.

Fig. 1: Scavenging of DPPH radicals. DPPH (50 $\mu \mathrm{M}$, upper panel and $100 \mu \mathrm{M}$, lower panel) was mixed with increasing concentrations of dihydroneopterin (-) or trolox (O) in Tris buffer containing $50 \%$ ethanol. After $20 \mathrm{~min}$ the absorbance at $517 \mathrm{~nm}$ was measured. The results of a typical experiment are shown.
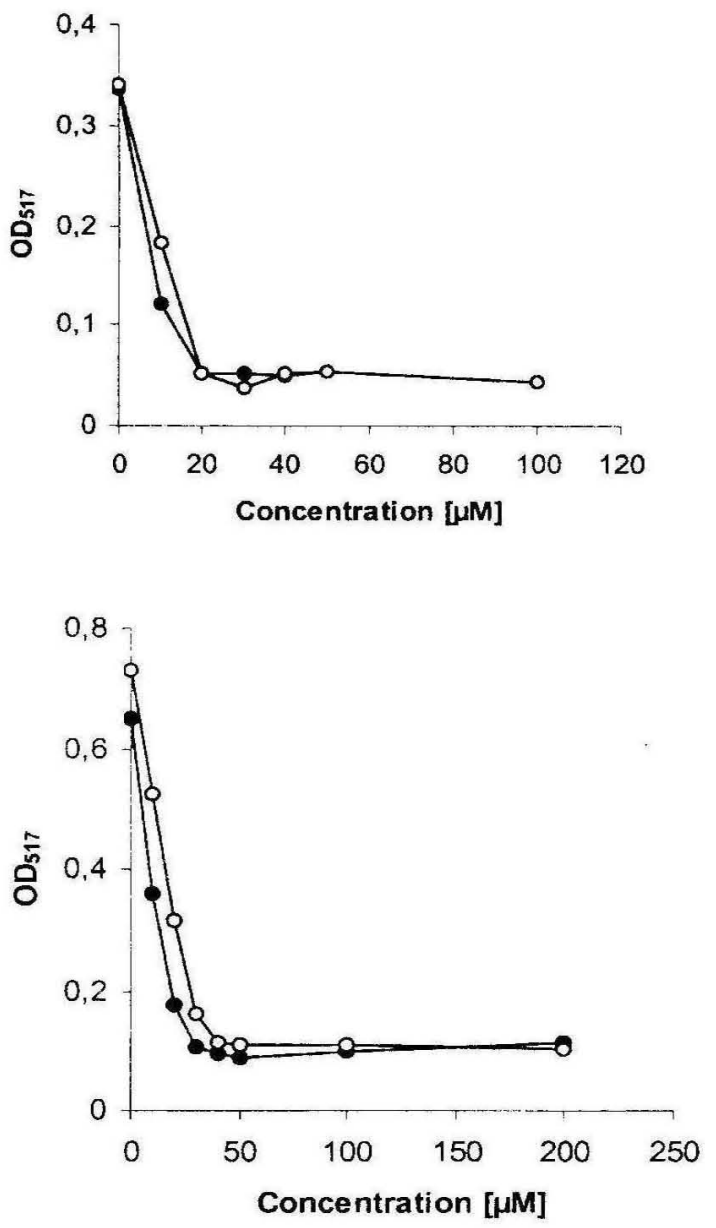

\section{Discussion}

Evidence is growing that pterins are involved in radical mediated reactions (1-17). However, a reliable view of the in vivo role of pterins concerning free radicals is hardly available. Although fully and partly reduced pterins have been described to be radical scavengers, these compounds are reducing agents and like ascorbate may act also prooxidatively in the presence of transition metal ions (15-17).

In this work we used two different nitrogen centered radicals, and we tested the ability of dihydroneopterin to scavenge these radicals. Both compounds are decolorized upon uptake of electrons and hence decolorization reflects the electron donating ability of the antioxidant used $(20,21,23,25)$. The concentration dependent scavenging of DPPH is essentially the same using dihydroneopterin and trolox, respectively. Both show maximum scavenging at approximately half the concentration of DPPH in 
the assay mixture. This means that both compounds donate two electrons per molecule. However, the reaction may proceed via different mechanisms using different solvents (24). In the ABTS assay we found the same rate of radical formation in the blank and in the mixture containing dihydroneopterin after the lag phase indicating that dihydroneopterin is not interfering with the radical generating system. This observation is important as radical

Fig. 2: Scavenging of ABTS radicals. Radical formation was achieved by the ABTS system in the absence of antioxidants $(\mathrm{O})$, in the presence of $26 \mu \mathrm{M}$ trolox (o) and in the presence of $8.2 \mu \mathrm{M}$ dihydroneopterin $(\bullet)$, respectively. Radical formation was followed by the absorbance at $620 \mathrm{~nm}$. Antioxidant activity is indicated by a lag phase in the formation of radicals (upper panel). In the lower panel the concentration dependence of the lag time for trolox (o) and dihydroneopterin (•) is shown.
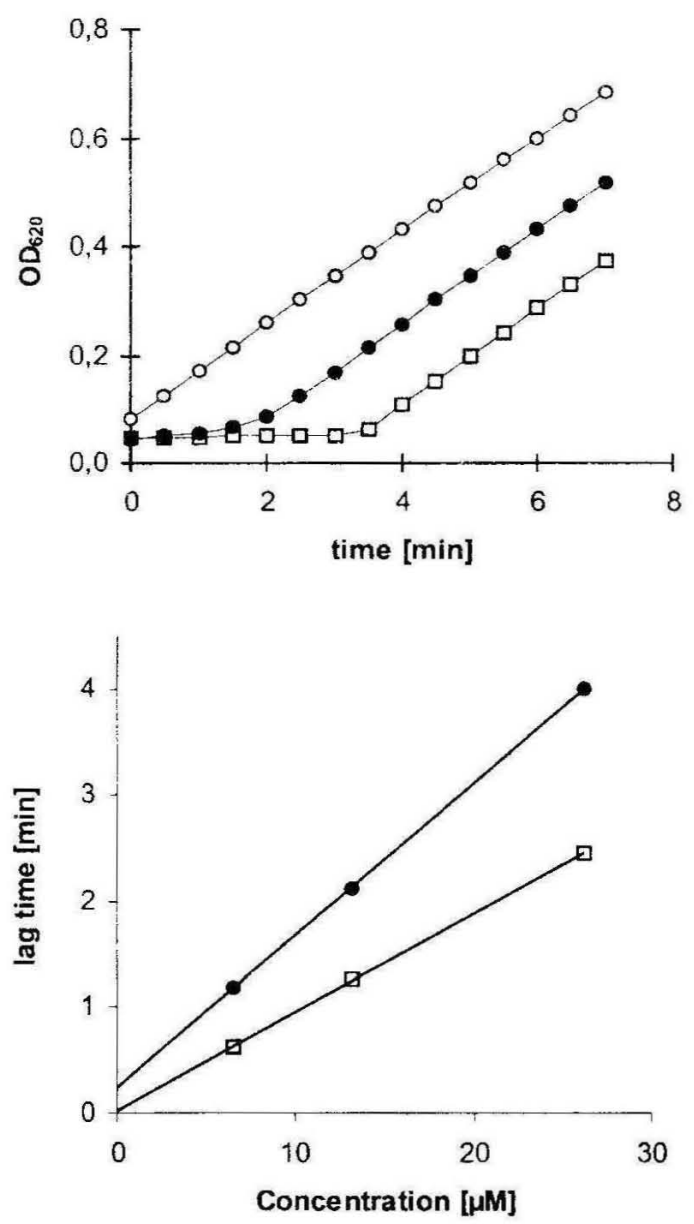

formation is catalyzed by metmyoglobin and interferences of dihydroneopterin with metaldependent radical formation has been demonstrated $(18,28)$. On the other hand the chelating ion cyanide was found to poisen the ABTS system without scavenging the formed radical (27). Similar to trolox the lag phase depends on the concentration of dihydroneopterin. However, the slope in the plot of lag time vs. concentration (Fig. 2, lower panel) is 50 $\%$ higher for dihydroneopterin (0.21) compared to trolox (0.14). This means if trolox donates two electrons dihydroneopterin donates three electrons to the $\mathrm{ABTS}^{+}$radical. The additional electron is not a contribution of the hydroxyl groups in the side chain of dihydroneopterin as neopterin has completely no effect on radical formation and the preformed radical in the ABTS system (data not shown).

From our data we cannot explain the differences in mechanism of decolorization of the $\mathrm{ABTS}^{+}$radical between dinydroneopterin and trolox. However, it is clearly denonstrated that in the used radical systems dihydroneopterin acts as an electron donating radical scavenger. The same property might be responsible for the prooxidative characteristics of dihydroneopterin in the presence of metal ions. We therefore speculate that the activity of dihydroneopterin concerning radical scavenging or promoting in a physiological environment similar to ascorbic acid depends st ongly on the presence of metal inns as.

\section{References}

1. Heales SJR, Blair J, A Meinschad C, Ziegler I. Inhibit on of monocyte luminol-dependent chemiluminescence by tetrahydrobiopterin, and the free radical oxidation of tetrahydrobiopterin, dihydrobioperin and dihydroneopterin. Cell Biochem Funct 1988 ; 6: 191-195.

2. Kojima S, Icho T, Kajiwara Y, Kubota K. Neopterin as an endogenous antioxidant. FEBS Lett 1992; 304: 163-166.

3. Weiss G, Fuchs D, Hausen, A, et al. Neopterin modulates toxicity mediated by reactive oxygen and chlorine species. FEBS Lett 1993; 321: 89-92.

4. Shen R. Inhibition of luminol-enhanced chemiluminescence by reduced pterins. Arch Biochem Biophys 1994; 310: 60-63.

5. Kojima S, Ona S, Iizuka I, Arai T, Mori H, Kubota K. Antioxidative activity of 5,6,7,8-tetrahydrobiopterin and its inhibitory effect on paraquat-induced cell toxicity in cultured rat hepatocytes. Free Rad Res 1995; 23: 419-430.

6. Gieseg S, Reibnegger G, Wachter H, Esterbauer, H. 7,8-dihydroneopterin inhibits low density lipoprotein oxidation in vitro. Evidence that this macrophage secreted pteridine is an anti-oxidant. Free Rad Res 1995; 23: 123-136.

7. Kojima S. Neopterin as an endogenous antioxidant.Pteridines 1995; 6: 181-184.

8. Baier-Bitterlich G, Fuchs D, Murr C et al. Effect of neopterin and 7,8-dihydroneopterin on tumor 
necrosis factor-a induced cell death, FEBS Lett 1995; 364: 234-238.

9. Reibnegger G, Fuchs D, Murr C, Dierich MP, Pfleiderer W, Wachter $\mathrm{H}$. Effects of pteridines on luminol-dependent chemiluminescence induced by chloramine-T. Free Rad Biol Med 1995; 18: 515 523.

10. Murr C, Baier-Bitterlich G, Fuchs D, et al. Effects of neopterin-derivatives on $\mathrm{H}_{2} \mathrm{O}_{2}$-induced luminol chemiluminescence: mechanistic aspects. Free Rad Biol Med 1996; 21: 449-456.

11. Horejsi R, Estelberger W, Mlekusch, W, et al. Effects of pteridines on chloramine-T-induced growth inhibition in E.coli strains: correlations with molecular structure. Free Rad Biol Med 1996; 21: $133-138$.

12. Oettl K, Dikalov S, Freisleben HJ, Mlekisch W, Reibnegger G. Spin trapping study of antioxidant properties of neopterin and 7,8-dihydroneopterw. Biochem Biophys Res Commun 1997; 234: 774778.

13. Widner B, Baier-Bitterlich G, Wede I, Wirleitner B, Wachter H, Fuchs D. Neopterin: Indicator of oxidative stress and part of the cytotoxic armature of activated macrophages in humans. Pteridines 1998; 9: 91-102.

14. Widner B, Baier-Bitterlich G, Wede I, Wirleitner, B, Fuchs D. Neopterin derivatives modulate the nitration of tyrosine by peroxynitrite. Biochchem Biophys Res Commun 1998; 248: 341-346.

15. Oettl K, Wirleitner B, Baier-Bitterlich G, Grammer T, Fuchs D, Reibnegger G. Formation of oxygen radicals in solutions of 7,8dihydroneopterin. Biochem Biophys Res Commun 1999; 264: 262-267.

16. Oettl K, Pfleiderer W, Reibnegger G. Formation of oxygen radicals in solutions of different 7,8dihydropterins: quantitative structure-activity relationships. Helv Chim Acta 2000; 83: 954-965.

17. Oettl K, Greilberger J, Reibnegger G. Dihydroneopterin and the generation of superoxide from iron ions. Pteridines 2000; in press

18. Regoli F, Winston GW. Quantification of total scavenging capacity of antioxidants for peroxynitrite, peroxyl radicals, and hydroxyl radicals. Toxicol Appl Pharm 1999; 156: 96-105.
19. Rice-Evans C, Miller NJ. Total antioxidant status in plasma and body fluids. Methods Enzymol 1994; 234: 279-293.

20. Begona Ruiz-Larrea M, Mohan AR, Pagnaga G, Miller NJ, Bolwell GP, Rice-Evans CA. Antioxidant activity of phytoestrogenic isoflavones. Free Rad Res 1997; 26: 63-70.

21. Rice-Evans CA, Miller NJ, Paganga G. Structureantioxidant activity relationships of flavonoids and phenolic acids. Free Radical Bio Med 1996; 20: 933-956.

22. Hatano T, Edamatsu R, Hiramatsu M, Mori A, Fujita Y, Yasuhara T, Yoshida T, Okuda T. Effects of the interaction of tannins with co-existing substances. VI. Effects of tannins and related polyphenols on superoxide anion radical, and on 1,1-diphenyl-2-picryhydrazyl radical. Chem Pharm Bull 1989; 37: 2016-2021.

23. Fauconneau B, Waffo-Teguo P, Huguet F, Barrier I, Decendit A, Merillon JM. Comparative study of radical scavenger and antioxidant propteries of phenolic compounds from vitis vinifera cell cultures using in vitro tests. Life Sci 1997; 21: 2103-2110.

24. Abe N, Nemoto A, Tsuchiya Y, Hojo H, Hirota A. Studies on the 1,1-diphenyl-2-picrylhydrazyl radical scavenging mechanism for a 2-pyrone compound. Biosci Biotechnol Biochem 2000; 64: 306-313.

25. Blois MS. Antioxidant determinatioins by the use of a stable free radical. Nature 1958; 181: 1199 1192.

26. Yamaguchi T, Takamura $H$, Matoba $T$, Terao J. HPLC method for evaluation of the free radicalscavenging activity of foods by using 1,1diphenyl-2-picrylhydrazyl. Biosci Biotechnol Biochem 1998; 62: 1201-1204.

27. Strube M, Haenen GRMM, Van den Berg H, Bast A. Pitfalls in a method for assessment of total antioxidant capacity. Free Rad Res 1997; 26: 515521.

28. Hausen A, Fuchs D, Reibnegger G, Werner ER, Werner-Felmayer $G$, Wachter $H$. Reduction of ferric iron by 7,8-dihydroneopterin. Pteridines 1990; $2: 83-85$. 